\title{
Wide-field optical detection of nanoparticles using on-chip microscopy and self-assembled nanolenses
}

\author{
Onur Mudanyali ${ }^{1,2, \dagger}$, Euan McLeod ${ }^{1,2, \dagger}$, Wei Luo ${ }^{1,2}$, Alon Greenbaum ${ }^{1,2}$, Ahmet F. \\ Coskun $^{1,2}$, Yves Hennequin ${ }^{3}$, Cédric P. Allier ${ }^{3}$, and Aydogan Ozcan ${ }^{1,2,4,5,{ }^{*}}$ \\ ${ }^{1}$ Electrical Engineering Department, University of California, Los Angeles, California 90095, USA \\ 2Bioengineering Department, University of California, Los Angeles, California 90095, USA \\ ${ }^{3}$ CEA, LETI, MINATEC, 17 rue des Martyrs, 38054 Grenoble cedex 9, France \\ ${ }^{4}$ California NanoSystems Institute, University of California, Los Angeles, California 90095, USA \\ ${ }^{5}$ Department of Surgery, David Geffen School of Medicine, University of California, Los Angeles, \\ California 90095, USA
}

\begin{abstract}
The direct observation of nanoscale objects is a challenging task for optical microscopy because the scattering from an individual nanoparticle is typically weak at optical wavelengths. Electron microscopy therefore remains one of the gold standard visualization methods for nanoparticles, despite its high cost, limited throughput and restricted field-of-view. Here, we describe a highthroughput, on-chip detection scheme that uses biocompatible wetting films to self-assemble aspheric liquid nanolenses around individual nanoparticles to enhance the contrast between the scattered and background light. We model the effect of the nanolens as a spatial phase mask centred on the particle and show that the holographic diffraction pattern of this effective phase mask allows detection of sub-100 nm particles across a large field-of-view of $>20 \mathrm{~mm}^{2}$. As a proof-of-concept demonstration, we report on-chip detection of individual polystyrene nanoparticles, adenoviruses and influenza A (H1N1) viral particles.
\end{abstract}

Nanoscale objects are difficult to visualize directly using optical techniques because of their small size compared to the optical wavelength, resulting in a weak scattering signal from individual nanoparticles. To mitigate this challenge, various techniques ${ }^{1-20}$ have been used for imaging sub-100 nm particles and viruses, including near-field optical microscopy ${ }^{1-4}$, super-resolution microscopy ${ }^{5-8,19,20}$, atomic force microscopy ${ }^{9,10}$, electron microscopy ${ }^{11-14}$ and other recently developed imaging techniques ${ }^{15-18}$. While generally providing excellent resolution, all of these existing approaches for imaging individual nanoparticles are

\footnotetext{
(c) 2013 Macmillan Publishers Limited. All rights reserved.

*Correspondence and requests for materials should be addressed to A.O., ozcan@ucla.edu.

$\dagger$ These authors contributed equally to this work.

Author contributions

O.M. performed the experiments and processed the resulting data. E.M. developed the theory and conducted numerical simulations and the related analysis. E.M., W.L., A.G and A.F.C. assisted in conducting the experiments and data analysis. O.M., E.M., Y.H., C.P.A. and A.O. planned the research and O.M., E.M. and A.O. wrote the manuscript.

A.O. supervised the project.

Supplementary information is available in the online version of the paper.

Reprints and permissions information is available online at www.nature.com/reprints.

Competing financial interests

Aydogan Ozcan is the co-founder of a start-up company that aims to commercialize lensfree microscopy tools.
} 
relatively bulky and low throughput, with a limited imaging field-of-view (FOV), typically less than $0.2 \mathrm{~mm}^{2}$ without mechanical scanning. These limitations pose challenges for the detection and enumeration of sparse nanoscale objects, such as viral particles at low concentrations. This can be particularly critical in early-stage disease diagnosis in point-ofcare settings or resource-limited environments, which ideally demand field-portable, costeffective and wide-field imaging and detection modalities.

Here, we introduce a compact, cost-effective and high-throughput optical microscopy technique that can detect individual sub- $100 \mathrm{~nm}$ particles and moderately sized viruses across an ultralarge FOV of, for example, $20.5 \mathrm{~mm}^{2}$ (Fig. 1a). Unlike some of the alternative techniques listed above, our technique does not circumvent the optical diffraction limit to boost spatial resolution and therefore cannot serve as a replacement for an ultrahighresolution microscopy modality, but instead it dramatically improves the signal-to-noise ratio (SNR) and contrast to enable detection of single nanoparticles over a very large FOV. At the heart of our approach lie two complementary techniques: (i) self-assembled liquid nanolenses and (ii) lensfree computational microscopy on a chip ${ }^{21-27}$. Note here that the term 'lensfree' refers to the lack of an 'imaging' lens or its equivalent between the sample and the sensor planes; that is, these self-assembled liquid nanolenses or the microlenses installed on each pixel of an optoelectronic sensor array are not considered as 'imaging' lenses. Using a sample preparation technique that only involves portable equipment and non-toxic chemicals, liquid nanolenses are assembled around each nanoparticle seated on a hydrophilic surface (Fig. 1b,c). These liquid lenses are composed of a biocompatible buffer that is stable for more than an hour at room temperature without significant evaporative loss. This buffer helps to prevent nanoparticle aggregation while also acting as a spatial phase mask that relatively enhances the lensfree diffraction signature of the embedded nanoparticle/nanolens assembly, as explained by our fluid and optical models of the system. Liquid film coatings with different compositions and sample preparation methods have been previously used in conjunction with optical microscopy; however, these earlier methods used thick (for example, $\sim 1 \mu \mathrm{m}$ ) and continuous films rather than isolated nanolenses selfassembled around individual particles, as a result of which they could not detect single nanoparticles smaller than $\sim 0.5-1 \mu \mathrm{m}$ in width or diameter ${ }^{21,28}$.

One of the most appropriate optical techniques to image such nanoscale objects is holography, because it converts the phase information into intensity oscillations through a heterodyne detection gain. We therefore used partially coherent lensfree digital in-line holography ${ }^{21-27,29-35}$ to sample the interference between the unscattered background light and the far-field diffraction patterns of individual nanolens/nanoparticle complexes using an optoelectronic sensor array (Fig. 1a). Because this platform operates under unit magnification without the use of any imaging lenses, its object FOV is equal to the active area of the sensor array, easily reaching, for example, $20-30 \mathrm{~mm}^{2}$ using the state-of-the-art complementary metal oxide semiconductor (CMOS) imaging chips that are now commonplace in modern cellphones ${ }^{26,27}$, or $10-20 \mathrm{~cm}^{2}$ with charge-coupled device (CCD) imaging chips (see, for example, Supplementary Figs S1,S2). The native resolution of these optoelectronic chips and the SNR of holographically reconstructed images can be further improved by pixel super-resolution techniques ${ }^{19,20,36-38}$. Here, we implement pixel superresolution by source shifting $23,24,26$, as illustrated in Fig. 1a. This technique captures several lensfree diffraction holograms from the same nano-particle, where the partially coherent light source is shifted in small increments, on the order of, for example, $0.1 \mathrm{~mm}$. A single pixel super-resolved holographic image is then synthesized from these sub-pixel shifted holograms, and is finally reconstructed to yield phase and amplitude images of the individual nanoparticles together with their surrounding self-assembled liquid lenses. 
These self-assembled nanolenses are generated using a sample preparation procedure that is described in Fig. 1c. In our experiments, we worked with concentrated nanoparticle solutions (polystyrene beads, Corpuscular), as well as cultured influenza A (H1N1) viral particles and adenoviruses, diluted in $0.1 \mathrm{M}$ Tris- $\mathrm{HCl}$ with $10 \%$ polyethylene glycol (PEG) 600 buffer (Sigma Aldrich). We then prepared a hydrophilic substrate by plasma-treating a glass coverslip using a portable and lightweight plasma generator for $\sim 5 \mathrm{~min}$, which is a critical step in enabling self-assembled liquid nanolens formation.

Immediately after plasma treatment, a 5-10 $\mu 1$ droplet of the dilute nanobead/virus suspension was transferred to the centre of the hydrophilic glass substrate (Fig. 1c,i). The sample was held flat for $3 \mathrm{~min}$ to allow for partial sedimentation of the nanoparticles, and then tilted with a slope of $3-5^{\circ}$ so that gravity slowly drove the droplet towards the edge of the substrate with an average speed of less than $1 \mathrm{~mm} \mathrm{~s}^{-1}$ (Fig. 1c,ii). A sparse monolayer of nanoobjects with surrounding liquid lenses remained in the wake of the droplet (Fig. 1c,iii). Once the droplet reached the edge of the substrate, the excess fluid was removed by tilting the sample at an angle of $15-20^{\circ}$ (Fig. 1c,iv). Following this last step, the nanoparticle/virus sample was then flipped through $180^{\circ}$ and placed onto a CMOS sensor array for lensfree holographic image acquisition (Fig. 1a and c,v). At this point, the remaining fluid volume in each nanolens was so small that its three-dimensional geometry was mainly determined by surface tension, making the effect of gravity negligible. In other words, this final $180^{\circ}$ rotation step does not affect the nanolens geometry. This entire sample preparation process takes less than $10 \mathrm{~min}$ and is performed without the use of a cleanroom.

After sample preparation, lensfree holographic image acquisition was performed using either only the green pixels of a 16 megapixel colour (RGB) CMOS chip or a large-area monochrome CCD chip (Supplementary Figs S1,S2). The sample was illuminated with a quasi-monochromatic fibre-coupled light source located at $Z_{1}=8-12 \mathrm{~cm}$ above the sensor array, as shown in Fig. 1a. For further miniaturization and field portability, the light source can also be a single light-emitting diode (LED) or an array of LEDs, enabling a compact microscopy architecture as demonstrated in our earlier work ${ }^{22,23,26}$. Because of the small object-to-sensor distance, as shown in Fig. $1 \mathrm{a}\left(Z_{2} \approx 300 \mu \mathrm{m}\right)$, the spatial coherence, temporal coherence and illumination alignment requirements for our microscopy setup are all relaxed, significantly reducing the speckle and multiple reflection noise artefacts over the entire active area of the CMOS array. On the other hand, because of unit magnification and the finite CMOS pixel size $(1.12 \mu \mathrm{m})$, individual lensfree holograms are undersampled, partially limiting the achievable spatial resolution and SNR. To mitigate this limitation, we implemented a pixel super-resolution technique, which digitally merges multiple holographic images that are shifted with respect to one another by sub-pixel pitch distances into a single high-resolution image ${ }^{23,24}$. Discrete source shifts of $\sim 0.1 \mathrm{~mm}$ translate to submicrometre hologram shifts at the detector plane due to the large $Z_{1}$ to $Z_{2}$ ratio of $>200$. These pixel super-resolved high-resolution holograms were then used to digitally reconstruct the complex object field at the sample plane using iterative phase retrieval techniques to eliminate twin image noise and obtain higher SNR microscopic images of the sample ${ }^{23,24}$ (see Supplementary Methods). As shown in Figs 2 and 3 and Supplementary Fig. S1, this reconstruction process is robust even at distant regions on the sensor array.

The effect of the number of holographic frames used for pixel super-resolution on the contrast and SNR of our nanoparticle images is characterized in the lower set of panels in Fig. 3. In these experiments, various lensfree holographic images of $95 \mathrm{~nm}$ beads were reconstructed from pixel super-resolved images synthesized using, for example, 1, 4, 8, 16, 36 and 64 sub-pixel shifted holographic frames, respectively. Reconstruction of a single lensfree frame did not provide any satisfactory result for detection of these $95 \mathrm{~nm}$ particles, whereas increasing the number of holographic frames used in our pixel super-resolution 
algorithm significantly enhanced the contrast and the SNR of individual nanoparticles (Fig. 3). Similar results were also obtained for $198 \mathrm{~nm}$ beads (Supplementary Fig. S7).

The combination of self-assembled liquid nanolenses and holographic computational onchip microscopy enables the detection of individual sub-100 nm particles (Figs 2-4) that are not visible with holographic imaging techniques alone, and have extremely low contrast even under conventional oil-immersion objective lenses (for example, with a numerical aperture (NA) of 1.25). As an example, we demonstrate in Fig. 4 that without the nanolenses, neither 198-nm- nor 95-nm-diameter polystyrene beads provide a signal above the background noise level in our lensfree holographic microscopy setup. However, with the formation of the above discussed nanolenses, these nanoparticles become clearly visible in both phase and amplitude reconstructions, as illustrated in Fig. 4 (Supplementary Figs S1S8). Both with and without the liquid lenses, the presence of the nanoparticles on the substrate is confirmed in these experiments using oil-immersion bright-field microscopy, although the contrast and SNR of these images are rather low despite the use of a highpower objective lens $(\times 100, \mathrm{NA}=1.25 ; \mathrm{Fig} .4)$. On the other hand, using lensfree on-chip microscopy, the contrast of the same nanoparticles is significantly improved (Fig. 4) after the formation of the nanolenses, which act as spatial phase masks enhancing the diffraction holograms of individual nanoparticles.

This contrast enhancement observed in our experiments is also supported by our fluid and optical system models. To shed more light on our observations, we begin by modelling the shape of the nanolens meniscus around each nanoparticle using the Young-Laplace equation $^{39-41}$ :

$$
\Delta p=\rho g h-\gamma\left(\frac{1}{R_{1}}+\frac{1}{R_{2}}\right)
$$

where $\Delta p$ is the over-pressure within the meniscus, $\rho$ is the fluid density, $g$ is the gravitational acceleration constant, $h$ is the height of the meniscus, $\gamma$ is the surface tension and $1 / R_{1}$ and $1 / R_{2}$ are the curvatures of the meniscus along its two principal directions. The Young-Laplace equation holds in general at length scales greater than a few tens of nanometres; below this scale, additional forces such as dispersion, van der Waals, steric or electrostatic forces must also be taken into account ${ }^{41,42}$.

We can non-dimensionalize equation (1) by the characteristic pressure $\sqrt{\gamma \rho g}$, which presents the capillary length scale $\ell_{c}=\sqrt{\gamma /(\rho g)}$ :

$$
\frac{\Delta p}{\sqrt{\gamma \rho g}}=\frac{h}{\ell_{c}}-\ell_{c}\left(\frac{1}{R_{1}}+\frac{1}{R_{2}}\right)
$$

For water, $\ell_{\mathrm{c}} \approx 2 \mathrm{~mm}$, while for aqueous PEG solutions such as ours the surface tension can be a factor of two smaller over a wide range of concentrations with similar density, making the capillary length shorter but still of roughly millimetre length ${ }^{43}$. The overpressure in the film, $\Delta p$, is coupled to the volume of the fluid surrounding the nanoparticle, and is determined by the formation process of the liquid nanolenses. As the fluid slowly drains due to the $<5^{\circ}$ tilt applied during sample preparation, the sparse nanoparticles pin the receding contact line until the surface tension of the fluid in contact with a nanoparticle can no longer support the hydrostatic pressure of the deformed contact line, at which point the fluidic bridge between the nanoparticle and the bulk receding contact line ruptures ${ }^{44-46}$. The maximum extent of the contact line deformation before rupture is on the order of the nanoparticle size ${ }^{47}$. Therefore the overpressure in the film immediately before and after 
rupture is on the order of $\rho g R_{\mathrm{p}}$, which makes $\Delta p / \sqrt{\gamma \rho g}$ of order $R_{\mathrm{p}} / \ell_{\mathrm{c}} \approx 1 \times 10^{-4}$. Note also that the gravitational term $h / \ell_{\mathrm{c}}$ is of the same order. However, the curvature terms are of order $\ell_{\mathrm{c}} / R_{\mathrm{p}} \approx 1 \times 10^{4}$. From this scaling analysis, we find that we are in the low Bond number limit where only the curvature terms are significant ${ }^{40}$. It is important to note that this approximation, $\Delta p \approx 0$, neglects the rapid rupture process, where the fluid bridge pinches off and additional overpressure may be introduced. However, quantifying this effect requires numerical fluid dynamic simulations ${ }^{47,48}$ that are beyond the scope of this manuscript. More importantly, with the $\Delta p \approx 0$ approximation, we find good agreement with our nanoparticle detection experiments.

Under these assumptions, the Young-Laplace equation (1) reduces to finding a surface with zero net curvature. In cylindrical coordinates, this can be written as ${ }^{40}$

$$
0=\frac{1}{R_{1}}+\frac{1}{R_{2}}=\frac{r^{\prime \prime}}{\left(1+r^{\prime 2}\right)^{3 / 2}}-\frac{1}{r \sqrt{1+r^{\prime 2}}}
$$

where $r=r(z)$ is the radial coordinate of the meniscus at an elevation $z$ above the substrate, and primes indicate derivatives with respect to $z$. The general solution to this nonlinear second-order ordinary differential equation can be written as a hyperbolic cosine:

$$
r(z)=\frac{1}{a b} \cosh [a(b z+1)]
$$

We refer to this last equation as the 'nanolens equation', which is used to determine the three-dimensional geometry of the self-assembled liquid lens around each nanoparticle. In this equation, $a$ and $b$ are constants that are determined by the contact angle at the particle $\left(\theta_{\mathrm{p}}\right)$, the contact angle at the substrate $\left(\theta_{\mathrm{s}}\right)$, as well as the particle radius $R_{\mathrm{p}}$ :

$$
\begin{gathered}
a=-\operatorname{arcsinh}\left(\cot \theta_{s}\right) \\
b=\frac{1}{z_{0}}\left[\frac{1}{a} \operatorname{arcsinh}\left(\frac{\beta\left(z_{0}\right) \cos \theta_{p}-\sin \theta_{p}}{\cos \theta_{p}+\beta\left(z_{0}\right) \sin \theta_{p}}\right)-1\right]
\end{gathered}
$$

where $z_{0}$ is the elevation of the meniscus-particle contact line and $\beta\left(z_{0}\right)$ is defined as

$$
\beta\left(z_{0}\right)=\frac{R_{p}-z_{0}}{\sqrt{R_{p}^{2}-\left(R_{p}-z_{0}\right)^{2}}}
$$

The elevation $z_{0}$ of the contact line can be determined by numerically solving the following transcendental equation derived from the intersection between the spherical particle surface and the meniscus shape, resulting in

$$
\left(\cos \theta_{p}+\beta\left(z_{0}\right) \sin \theta_{p}\right) \times\left[\operatorname{arcsinh}\left(\frac{\beta\left(z_{0}\right) \cos \theta_{p}-\sin \theta_{p}}{\cos \theta_{p}+\beta\left(z_{0}\right) \sin \theta_{p}}\right)-a\right]=\frac{R_{p}}{2 R_{p}-z_{0}}
$$

The particle diameter $R_{\mathrm{p}}$ linearly scales with both the height and lateral extent of the meniscus, but does not affect its shape or aspect ratio. Although both $\theta_{\mathrm{s}}$ and $\theta_{\mathrm{p}}$ influence all 
aspects of the meniscus shape, $\theta_{\mathrm{s}}$ most significantly affects the radial extent of the meniscus, while $\theta_{\mathrm{p}}$ moderately affects its thickness.

Some representative solutions of nanolens equation (4) for different contact angles are shown in Fig. 1b,i-iii. The measured contact angle of a $\sim 1$-mm-radius droplet on a plasmatreated glass coverslip is $\theta_{\mathrm{s}}=10^{\circ}$, and the measured contact angle on a polystyrene surface is $\theta_{\mathrm{p}}=50^{\circ}$. We use these macroscopic contact angles as nominal values for the microscopic system in Fig. 1b,i as we cannot directly measure the contact angles at our size scale. Small variations in contact angles can affect the aspect ratio of the meniscus, as illustrated in Fig. $1 b$,ii and iii, but do not alter its general shape. The scanning electron microscopy (SEM) image shown in Fig. 1b,iv is typical of the nanolens after it has been desiccated by the vacuum required in SEM sample preparation. Although the original shape of the liquid film has not been preserved because of the vacuum, it is clear that the liquid residue from the film only extends a distance on the order of the particle diameter, in good agreement with our model predictions (for example, see the curve in Fig. 1b,iv).

To evaluate the optical effects of each nanolens on the recorded lensfree holograms of the nanoparticles, we use two numerical models: (i) a finite-difference time-domain (FDTD) simulation followed by Rayleigh-Sommerfeld wave propagation and (ii) a thin-lens model followed by Rayleigh-Sommerfeld wave propagation. In the FDTD model (Fig. 1b) we simulate the particle $\left(n_{\mathrm{p}}=1.61\right)^{49}$, the nanolens $\left(n_{\mathrm{f}}=1.35\right)^{50}$ and the substrate $\left(n_{\mathrm{s}}=1.52\right)$ within a simulation volume of $20 \times 20 \times 5 \mu \mathrm{m}^{3}$, calculating the amplitude and phase of the transmitted optical field $3 \mu \mathrm{m}$ beyond the glass-air interface; that is, no evanescent waves are considered as our detection occurs beyond the near-field. These results are then substituted at the centre of a larger $\left(100 \times 100 \mu \mathrm{m}^{2}\right)$ homogeneous field (that is, uniform plane wave) that is numerically propagated a distance of $297 \mu \mathrm{m}\left(Z_{2}-3 \mu \mathrm{m}\right)$, resulting in a simulated lensfree diffraction hologram. In the thin lens model, however, we ignore threedimensional scattering and represent the particle and its surrounding nanolens as a single two-dimensional phase-only object whose phase delay as a function of the radial coordinate is the free-space wavenumber $k_{0}$ times the line integral of the optical path length in $z$ through the entire depth of the materials at that coordinate (Supplementary Fig. S9). For both of these optical models, the nanolens equation (4), described above, is used to estimate the three-dimensional geometry of the liquid lens that forms around each nanoparticle.

To provide a fair comparison to our experimental results, we downsample the numerically generated lensfree holograms to a super-resolved effective pixel size (that is, $0.28 \mu \mathrm{m}$ ), then add randomly generated Gaussian noise to each hologram and quantize the pixel values to 10-bit levels. In Fig. 5, these numerically generated noisy holograms are used to attempt to reconstruct $95 \mathrm{~nm}$ particles with and without nanolenses. For both the FDTD and thin-lens models, the nanolenses significantly improve the image contrast such that the nanoparticle can be clearly distinguished from the background noise in both the amplitude and phase reconstructions. Without the liquid nanolens, however, the same numerical models reveal that the signature of the $95 \mathrm{~nm}$ particle is effectively lost within the background noise (Fig. 5), also agreeing with our experimental observations in Fig. 4. A more detailed comparison between the experimental and theoretical nanolens phase functions is illustrated in Supplementary Fig. S10, which provides an independent verification of the nanolens shape predicted by our theoretical analysis. Furthermore, a numerical study of the effects of the nanolens film properties (such as refractive index, extinction coefficient, substrate contact angle) is also presented in Supplementary Fig. S11, shedding more light on the detection limits of our approach.

Finally, using the same lensfree platform we demonstrated computational on-chip detection of single H1N1 virus particles and sub-100 nm adenoviruses (Fig. 6). Different super- 
resolved holographic regions of interest were digitally cropped from a much larger FOV $\left(20.5 \mathrm{~mm}^{2}\right)$ for these virus samples, and were then digitally reconstructed to yield both lensfree amplitude and phase images of the viral particles. For comparison, $\times 100$ oilimmersion objective (NA $=1.25)$ and SEM images of the same samples are also shown, which match our reconstructed images very well. For the particularly small adenoviruses, we find phase reconstructions perform better than amplitude images, as they exhibit greater SNR and contrast.

In conclusion, we have introduced a compact, cost-effective and high-throughput computational on-chip microscopy technique that can detect individual sub-100 nm particles and viruses across an ultralarge FOV of $20.5 \mathrm{~mm}^{2}$, that is, more than two orders of magnitude larger than other nanoimaging techniques. Through a wetting film-based method that induces self-assembled liquid nanolenses around individual particles, we reconstruct both amplitude and phase images of single nanoparticles that are otherwise undetectable with on-chip microscopy. The enhancement provided by the self-assembled nanolenses is well understood through analytical models of the liquid meniscus shape and numerical models of its contribution to the optical diffraction signal.

\section{Methods}

\section{Sample preparation}

Samples were received as concentrated nanoparticle solutions (polystyrene beads, Corpuscular) as well as cultured influenza A (H1N1) viral particles and adenoviruses, which were fixed using $1.5 \%$ formaldehyde. The virus specimens, with an initial density of $100,000 \mu \mathrm{l}^{-1}$, were centrifuged at $\sim 25,000 \mathrm{~g}$, and the supernatant was separated and filtered using a syringe filter with a pore size of $0.2 \mu \mathrm{m}$ to remove larger contaminations and clusters. Small volumes of concentrated nanobead or virus solutions were then diluted at room temperature using 0.1 M Tris-HCl with 10\% PEG 600 buffer (Sigma Aldrich), and were sonicated for $\sim 2 \mathrm{~min}$ so that the final concentration was $>20,000 \mu \mathrm{l}^{-1}$. We then prepared a hydrophilic substrate by cleaning a $22 \mathrm{~mm} \times 22 \mathrm{~mm}$ glass coverslip (Fisher Scientific) with isopropanol and distilled water, then plasma-treating it using a portable and lightweight plasma generator (Electro-Technic Products, BD-10AS) for $\sim 5 \mathrm{~min}$.

\section{Image acquisition}

Lensfree holographic image acquisition was performed using only the green pixels of a 16megapixel colour (RGB) CMOS chip (Sony) or a monochrome 39-megapixel CCD chip (Kodak). The sample was illuminated with a quasi-monochromatic light source with a central wavelength of $480 \mathrm{~nm}$ and a spectral bandwidth of $\sim 3 \mathrm{~nm}$, coupled to a multimode fibre (core size, $0.1 \mathrm{~mm}$ ), the end of which was located at $Z_{1}=8-12 \mathrm{~cm}$ above the sensor array, as shown in Fig. 1a.

\section{Image processing}

For details regarding image processing, see Supplementary section, 'Computational Methods'.

\section{Supplementary Material}

Refer to Web version on PubMed Central for supplementary material. 


\section{Acknowledgments}

Ozcan Research Lab acknowledges the support of the Army Research Office Young Investigator Award, the Presidential Early Career Award for Scientists and Engineers (PECASE), an NSF CAREER Award, an Office of Naval Research Young Investigator Award and the National Institutes of Health (NIH) Director's New Innovator Award (DP2OD006427) from the Office of The Director, NIH. The work at CEA-Leti was supported by the Carnot Institutes Network. The authors thank Hangfei Qi and Ren Sun of UCLA for H1N1 and adenovirus specimens.

\section{References}

1. Betzig E, Chichester RJ. Single molecules observed by near-field scanning optical microscopy. Science. 1993; 262:1422-1425. [PubMed: 17736823]

2. Lange FD, et al. Cell biology beyond the diffraction limit: near-field scanning optical microscopy. J Cell Sci. 2001; 114:4153-4160. [PubMed: 11739648]

3. Kalkbrenner T, Ramstein M, Mlynek J, Sandoghdar V. A single gold particle as a probe for apertureless scanning near-field optical microscopy. J Microsc. 2001; 202:72-76. [PubMed: 11298873]

4. Ozcan A, et al. Differential near-field scanning optical microscopy. Nano Lett. 2006; 6:2609-2616. [PubMed: 17090100]

5. Huang FM, Zheludev NI. Super-resolution without evanescent waves. Nano Lett. 2009; 9:12491254. [PubMed: 19182908]

6. Huang B, Babcock H, Zhuang X. Breaking the diffraction barrier: super-resolution imaging of cells. Cell. 2010; 143:1047-1058. [PubMed: 21168201]

7. Kner P, Chhun BB, Griffis ER, Winoto L, Gustafsson MGL. Super-resolution video microscopy of live cells by structured illumination. Nature Methods. 2009; 6:339-342. [PubMed: 19404253]

8. Fitzgibbon J, Bell K, King E, Oparka K. Super-resolution imaging of plasmodesmata using threedimensional structured illumination microscopy. Plant Physiol. 2010; 153:1453-1463. [PubMed: 20508140]

9. Binnig G, Quate CF, Gerber C. Atomic force microscope. Phys Rev Lett. 1986; 56:930-933. [PubMed: 10033323]

10. Ebenstein Y, Nahum E, Banin U. Tapping mode atomic force microscopy for nanoparticle sizing: tip-sample interaction effects. Nano Lett. 2002; 2:945-950.

11. Chithrani BD, Ghazani AA, Chan WCW. Determining the size and shape dependence of gold nanoparticle uptake into mammalian cells. Nano Lett. 2006; 6:662-668. [PubMed: 16608261]

12. Feinstone SM, Kapikian AZ, Purcell RH. Hepatitis A: detection by immune electron microscopy of a virus-like antigen associated with acute illness. Science. 1973; 182:1026-1028. [PubMed: 4356028]

13. Hockley DJ, Wood RD, Jacobs JP, Garrett AJ. Electron microscopy of human immunodeficiency virus. J Gen Virol. 1988; 69(10):2455-2469. [PubMed: 2459300]

14. Pease LF III, et al. Quantitative characterization of virus-like particles by asymmetrical flow field flow fractionation, electrospray differential mobility analysis, and transmission electron microscopy. Biotechnol Bioeng. 2009; 102:845-855. [PubMed: 18958863]

15. Shevchuk AI, et al. Imaging single virus particles on the surface of cell membranes by highresolution scanning surface confocal microscopy. Biophys J. 2008; 94:4089-4094. [PubMed: 18199668]

16. Ignatovich FV, Topham D, Novotny L. Optical detection of single nanoparticles and viruses. IEEE J Sel Top Quantum Electron. 2006; 12:1292-1300.

17. Daaboul GG, et al. High-throughput detection and sizing of individual low-index nanoparticles and viruses for pathogen identification. Nano Lett. 2010; 10:4727-4731. [PubMed: 20964282]

18. Hong $X$, et al. Background-free detection of single $5 \mathrm{~nm}$ nanoparticles through interferometric cross-polarization microscopy. Nano Lett. 2011; 11:541-547. [PubMed: 21204579]

19. Stern A, Javidi B. Improved-resolution digital holography using the generalized sampling theorem for locally band-limited fields. J Opt Soc Am A. 2006; 23:1227-1235. 
20. Rivenson Y, Stern A, Javidi B. Single exposure super-resolution compressive imaging by double phase encoding. Opt Express. 2010; 18:15094-15103. [PubMed: 20639994]

21. Mudanyali O, Bishara W, Ozcan A. Lensfree super-resolution holographic microscopy using wetting films on a chip. Opt Express. 2011; 19:17378-17389. [PubMed: 21935102]

22. Mudanyali O, et al. Compact, light-weight and cost-effective microscope based on lensless incoherent holography for telemedicine applications. Lab on a Chip. 2010; 10:1417-1428. [PubMed: 20401422]

23. Bishara W, et al. Holographic pixel super-resolution in portable lensless on-chip microscopy using a fiber-optic array. Lab Chip. 2011; 11:1276-1279. [PubMed: 21365087]

24. Bishara W, Su T-W, Coskun AF, Ozcan A. Lensfree on-chip microscopy over a wide field-of-view using pixel super-resolution. Opt Express. 2010; 18:11181-11191. [PubMed: 20588977]

25. Isikman SO, et al. Lens-free optical tomographic microscope with a large imaging volume on a chip. Proc Natl Acad Sci USA. 201110.1073/pnas.1015638108

26. Greenbaum A, Sikora U, Ozcan A. Field-portable wide-field microscopy of dense samples using multi-height pixel super-resolution based lensfree imaging. Lab Chip. 2012; 12:1242-1245. [PubMed: 22334329]

27. Tseng D, et al. Lensfree microscopy on a cellphone. Lab Chip. 2010; 10:1787-1792. [PubMed: 20445943]

28. Allier CP, Hiernard G, Poher V, Dinten JM. Bacteria detection with thin wetting film lensless imaging. Biomed Opt Express. 2010; 1:762-770. [PubMed: 21258507]

29. Gopinathan U, Pedrini G, Javidi B, Osten W. Lensless 3D digital holographic microscopic imaging at vacuum UV wavelength. J Display Technol. 2010; 6:479-483.

30. Marquet P, et al. Digital holographic microscopy: a noninvasive contrast imaging technique allowing quantitative visualization of living cells with subwavelength axial accuracy. Opt Lett. 2005; 30:468-470. [PubMed: 15789705]

31. Dubois F, Joannes L, Legros J-C. Improved three-dimensional imaging with a digital holography microscope with a source of partial spatial coherence. Appl Opt. 1999; 38:7085-7094. [PubMed: 18324255]

32. Moon I, Javidi B. 3-D visualization and identification of biological microorganisms using partially temporal incoherent light in-line computational holographic imaging. IEEE Trans Med Imag. 2008; 27:1782-1790.

33. Xu W, Jericho MH, Meinertzhagen IA, Kreuzer HJ. Digital in-line holography for biological applications. Proc Natl Acad Sci USA. 2001; 98:11301-11305. [PubMed: 11572982]

34. Javidi B, Yeom S, Moon I, Daneshpanah M. Real-time automated 3D sensing, detection, and recognition of dynamic biological micro-organic events. Opt Express. 2006; 14:3806-3829. [PubMed: 19516528]

35. Javidi B, Moon I, Yeom S, Carapezza E. Three-dimensional imaging and recognition of microorganism using single-exposure on-line (SEOL) digital holography. Opt Express. 2005; 13:4492-4506. [PubMed: 19495364]

36. Zalevsky Z, Gur E, Garcia J, Micó V, Javidi B. Superresolved and field-of-view extended digital holography with particle encoding. Opt Lett. 2012; 37:2766-2768. [PubMed: 22743522]

37. Borkowski A, Zalevsky Z, Javidi B. Geometrical superresolved imaging using nonperiodic spatial masking. J Opt Soc Am A. 2009; 26:589-601.

38. Mendlovic, D.; Zalevsky, Z. Optical Super Resolution. Erich Schmidt; 2003.

39. Young T. An essay on the cohesion of fluids. Phil Trans R Soc Lond. 1805; 95:65-87.

40. Gennes, P-GD.; Brochard-Wyart, F.; Quéré, D. Capillarity and Wetting Phenomena: Drops, Bubbles, Pearls, Waves. Springer; 2004.

41. Yeh EK, Newman J, Radke CJ. Equilibrium configurations of liquid droplets on solid surfaces under the influence of thin-film forces: Part II. Shape calculations. Colloids Surf. 1999; 156:525546.

42. Israelachvili, JN. Intermolecular and Surface Forces. Academic Press; 2011. 
43. Compostizo A, Cancho SM, Rubio RG, Crespo Colin A. Experimental study of the equation of state and the surface tension of water-soluble polymers: poly(ethylene glycol)- $b$-poly(propylene glycol)- $b$-poly(ethylene glycol) + water at 298.15 K. Phys Chem Chem Phys. 2001; 3:1861-1866.

44. Nadkarni GD, Garoff S. An investigation of microscopic aspects of contact angle hysteresis: pinning of the contact line on a single defect. Europhys Lett. 1992; 20:523-528.

45. Marsh JA, Cazabat AM. Dynamics of contact line depinning from a single defect. Phys Rev Lett. 1993; 71:2433-2436. [PubMed: 10054679]

46. Born P, Blum S, Munoz A, Kraus T. Role of the meniscus shape in large-area convective particle assembly. Langmuir. 2011; 27:8621-8633. [PubMed: 21699167]

47. Bonn D, Eggers J, Indekeu J, Meunier J, Rolley E. Wetting and spreading. Rev Mod Phys. 2009; 81:739-805.

48. Beltrame P, Knobloch E, Hänggi P, Thiele U. Rayleigh and depinning instabilities of forced liquid ridges on heterogeneous substrates. Phys Rev E. 2011; 83:016305.

49. Kasarova SN, Sultanova NG, Ivanov CD, Nikolov ID. Analysis of the dispersion of optical plastic materials. Opt Mater. 2007; 29:1481-1490.

50. Mohsen-Nia M, Modarress H, Rasa H. Measurement and modeling of density, kinematic viscosity, and refractive index for poly(ethylene glycol) aqueous solution at different temperatures. J Chem Eng Data. 2005; 50:1662-1666. 


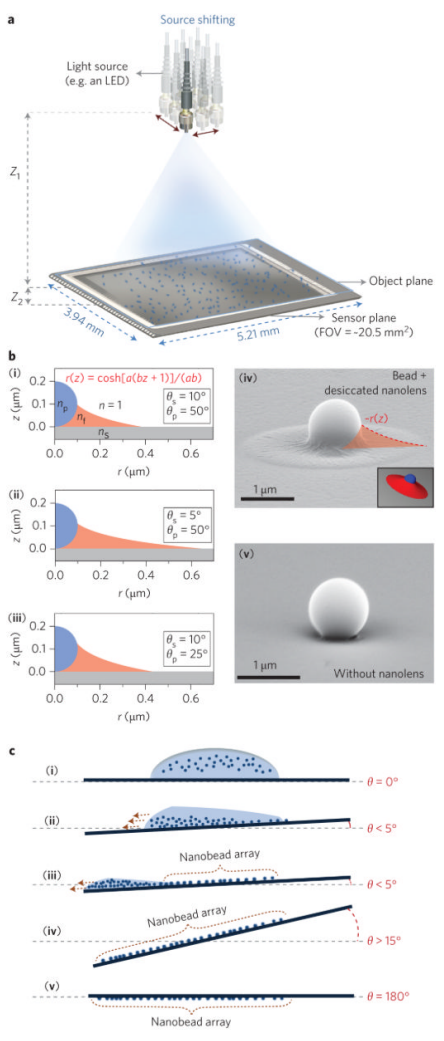

Figure 1. Experimental setup, numerical model and sample preparation

a, Lensfree pixel super-resolution holography schematic $\left(Z_{1} \approx 8-12 \mathrm{~cm}, Z_{2} \approx 300 \mu \mathrm{m}\right)$. Discrete lateral shifts of the fibre-coupled source create sub-pixel $(<1.12 \mu \mathrm{m})$ image shifts on the detector plane, which are used to generate a pixel super-resolved holographic image. b, Self-assembled liquid nanolens (meniscus) shapes for different substrate $\left(\theta_{\mathrm{s}}\right)$ and particle $\left(\theta_{\mathrm{p}}\right)$ contact angles (i-iii), and SEM images of beads with (iv) and without $(\mathbf{v})$ desiccated residue from a nanolens. Inset to iv: three-dimensional model used in the optical simulations. c, Overview of the sample preparation technique. 


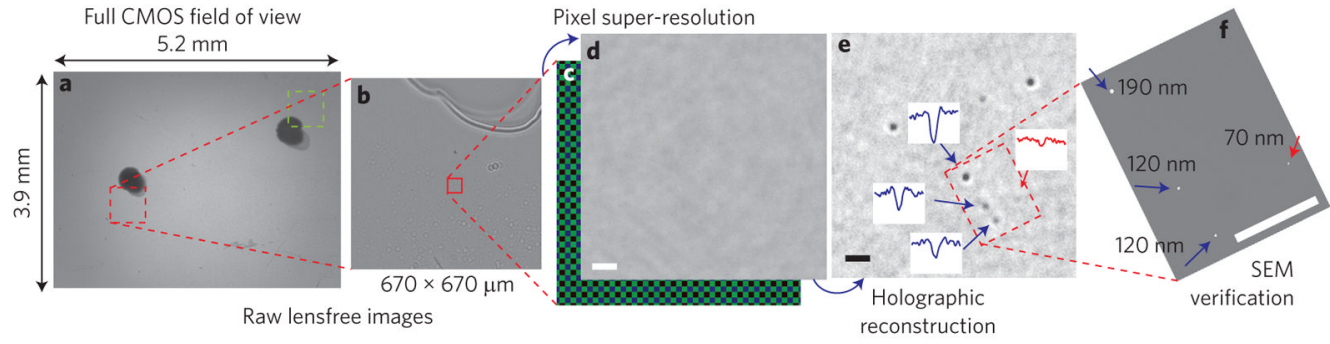

Figure 2. Large-FOV lensfree holographic microscopy of nanoparticles $\mathbf{a}, \mathbf{b}$, Full FOV of the CMOS chip (a) and a zoomed-in region (b). The large black marks in a facilitate registration with the SEM images. The green square in a is also shown, expanded, in Fig. 3. c,d, Raw lensfree Bayer-pattern RGB images (c) are converted into high-resolution monochrome holograms (d) via pixel super-resolution. e, Reconstructing the super-resolved holographic images enables the detection of single nanoparticles. $\mathbf{f}$, This is verified by SEM. Various other SEM FOVs and their lensfree reconstruction comparisons are included in Fig. 3 and Supplementary Figs S3-S6. Scale bars, $5 \mu \mathrm{m}$. 

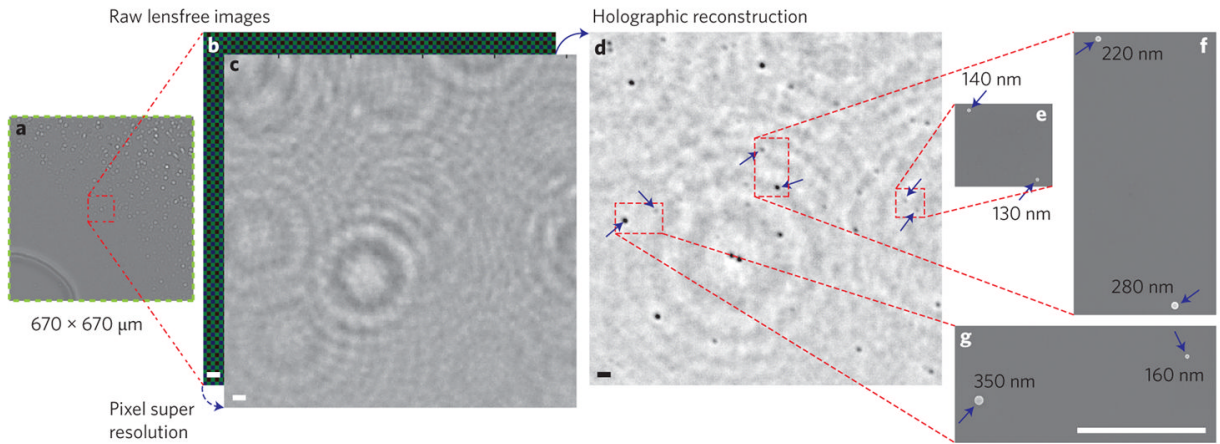

SEM verifications

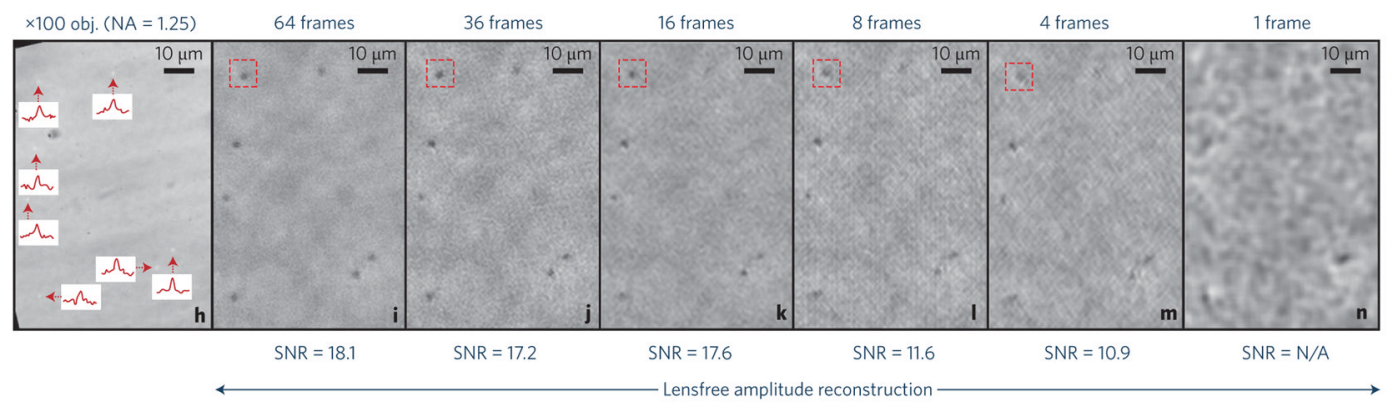

Figure 3. Lensfree holographic reconstructions of nanoparticles

a-g, Similar to Fig. 2b-f, the green square region from Fig. 2a is reconstructed to demonstrate the robustness of nanolens formation and lensfree holography across a large FOV (verified by SEM images). $\mathbf{h}-\mathbf{n}$, Demonstration of improvement in contrast and SNR of $95 \mathrm{~nm}$ particles using pixel super-resolution. With $\geq 16$ sub-pixel-shifted lensfree frames $(\mathbf{i}-\mathbf{k})$, individual nanoparticles are detectable using nanolenses. SNR values correspond to the $95 \mathrm{~nm}$ particle within the red square. For comparison (h), a bright-field, oil-immersion $\times 100$ objective lens $(\mathrm{NA}=1.25)$ image of the same sample is shown with intensity crosssections. Scale bars, $5 \mu \mathrm{m}(\mathbf{b}-\mathbf{d}, \mathbf{g})$; images in $\mathbf{e}-\mathbf{g}$ have the same scale. 
$198 \mathrm{~nm}$ beads (Experiment)
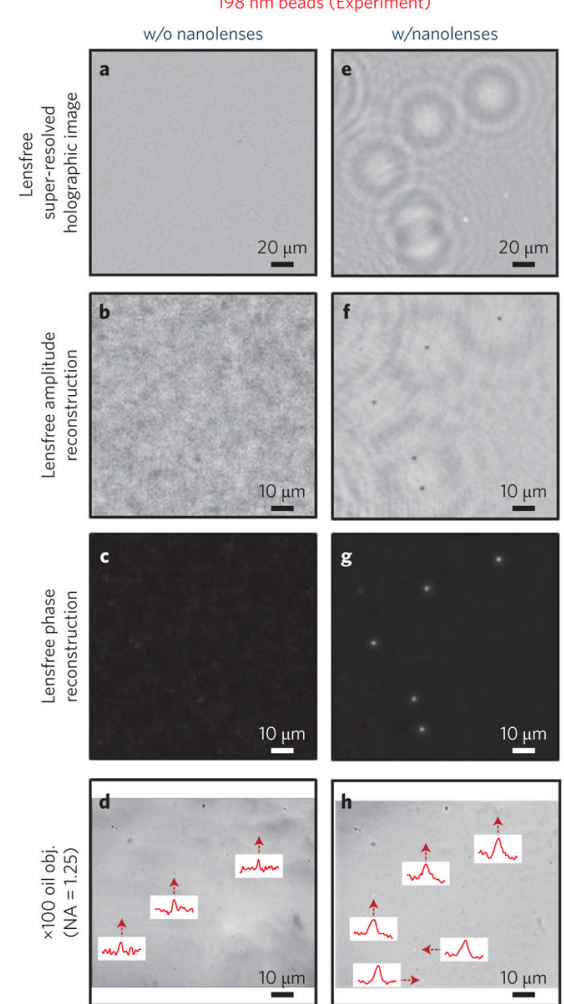

$95 \mathrm{~nm}$ beads (Experiment)

w/o nanolenses
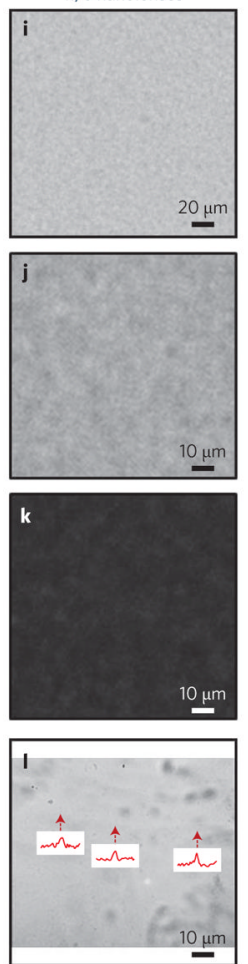

w/nanolenses
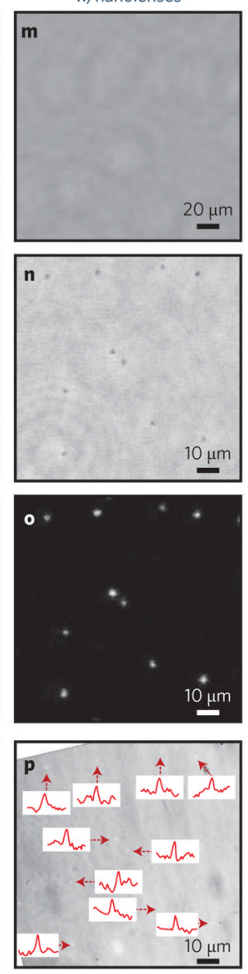

Figure 4. Lensfree holographic detection of $198 \mathrm{~nm}$ and $95 \mathrm{~nm}$ polystyrene beads with and without self-assembled nanolenses

Using lensfree microscopy, neither $198 \mathrm{~nm}(\mathbf{a}-\mathbf{d})$ nor $95 \mathrm{~nm}$ beads (i-l) can be detected using regular smears without nanolenses. In contrast, the formation of liquid nanolenses enables holographic detection of both bead sizes via amplitude and phase images (e-g,m-o). Nanolens-based lensfree holographic images (f,g,n,o) (cropped from a much larger FOV of $\left.>20 \mathrm{~mm}^{2}\right)$ are in good agreement with $\times 100$ oil-immersion objective lens $(\mathrm{NA}=1.25)$ images of the same samples $(\mathbf{h}, \mathbf{p})$, although the contrast is relatively poor in these brightfield images. 

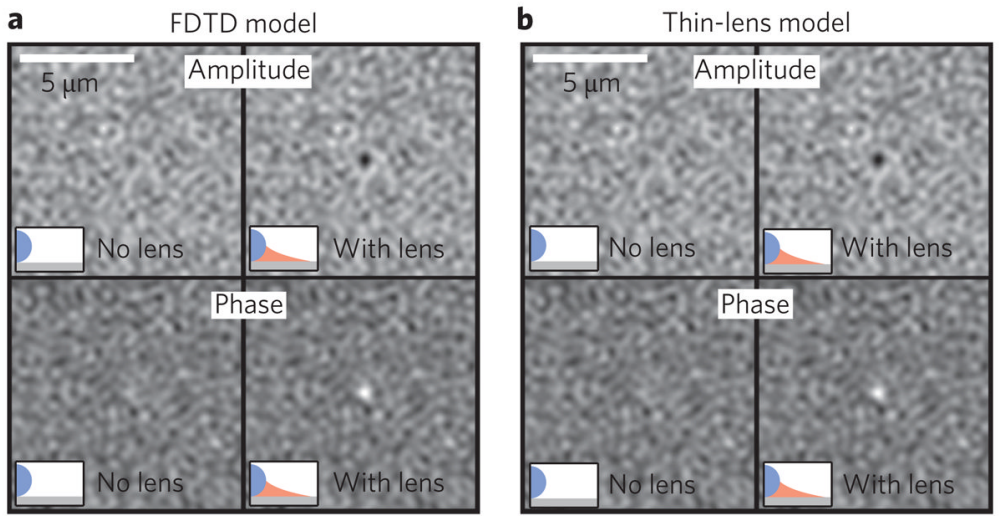

Figure 5. Simulated digital holographic reconstructions illustrating how liquid nanolenses enable the detection of otherwise undetectable $95 \mathrm{~nm}$ particles

a, The results of an FDTD simulation are holographically propagated and then reconstructed. b, The thin lens model is used to generate the holograms. In these simulations a super-resolved pixel size of $0.28 \mu \mathrm{m}$ is used. Both amplitude and phase reconstructions are shown in each case, with the nanoparticle located at the centre of the frame. The standard deviation of the Gaussian noise added to the lensfree holograms is $1 \%$ of the mean hologram intensity. 

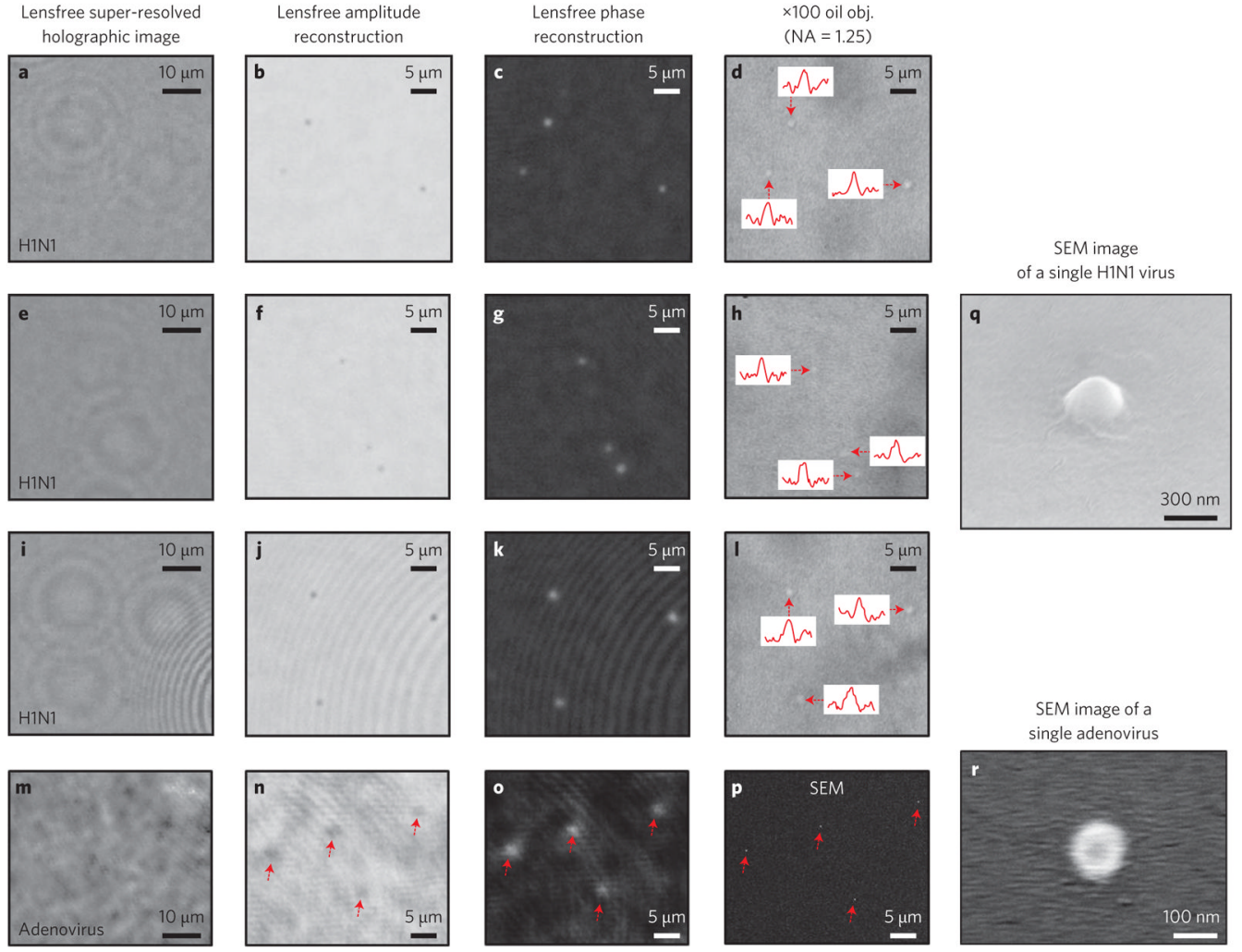

Figure 6. Detection of viruses

Lensfree pixel super-resolved holographic detection of individual influenza A (H1N1) viruses $(\mathbf{a}-\mathbf{c}, \mathbf{e}-\mathbf{g}, \mathbf{i}-\mathbf{k})$ and adenoviruses $(\mathbf{m}-\mathbf{o})$. Holographic fringes for adenoviruses are weak due to their smaller size $(<100 \mathrm{~nm})$. Bright-field oil-immersion objective lens $(\times 100$, $\mathrm{NA}=1.25)$ images of H1N1 samples are shown for comparison $(\mathbf{d}, \mathbf{h}, \mathbf{l}) . \mathbf{p}$, Because adenoviruses could not be observed under bright-field microscopy, SEM was instead used for their verification. q,r, A tilted SEM image of a single H1N1 virus surrounded by a liquid nanolens desiccated by SEM sample preparation is shown $(\mathbf{q})$, as is a normal-incidence SEM image of a single adenovirus $(\mathbf{r})$. 